\author{
Petruța Spânu \\ Alexandru Ioan Cuza University \\ Bulevardul Carol I 11, Iaşi 700506, Romania
}

\title{
Les Chardons du Baragan de Panaït Istrati, un récit de la faim
}

\begin{abstract}
The novel The Thistles of the Baragan (1928), the most important in the oeuvre of Panait Istrati (1884-1935), is not only an indictment against exploitation but also a story on hunger, inspired by the Romanian rural world of the early twentieth century, which summarizes adventures of the adolescent Matake. He will discover social inequality and poverty, and he will face both individual and collective death. He will see revolt everywhere and will be part of it. His long journey will last as long as his frustrated and traumatized childhood and will lead him to social meditation.
\end{abstract}

Qui est Panaït Istrati (1884-1935), dont on parle tellement aux années vingt du $\mathrm{XX}^{\mathrm{e}}$ siècle ? En quoi consiste son « cas »?

$\mathrm{Au}$ début, il y a une belle légende : un vagabond balkanique, un pauvre Roumain misérable, rongé par la misère et la tuberculose, déjà vieillissant, autodidacte, décidé à en finir avec la vie et tente de se suicider dans le jardin public de Nice le 3 janvier 1921, en se tranchant la gorge avec une lame de rasoir. Mais, au lieu de mourir anonyme, il est sauvé, et reconnu comme écrivain, dans deux littératures, française et roumaine, devient célèbre en France, puis en Europe, dans l'immense Russie bolchevique, ensuite outre-Atlantique. Dans les papiers du malheureux transporté à l'hôpital, on trouve deux lettres, l'une adressée au journal L'Humanité, expliquant son geste et 
s'achevant par un salut au "prolétariat français », et l'autre, envoyée deux ans plus tôt à Romain Rolland, et retournée avec la mention «parti sans adresse ». Il est sauvé, et la lettre arrive enfin au destinataire, qui découvre un génie littéraire en herbe. Il lui répond, l'exhorte à écrire, le guide, facilite la publication de son premier article dans la revue, puis de son premier roman, Kyra Kyralina (1923), dont il écrit la préface mémorable, «Un Gorki balkanique ». Ce roman ouvre le cycle des récits d'Adrien Zograffi. Ce nom, qui signifie « écrivain » (du grec ancien zôon «être vivant, animal» et de graphein «écrire »), dissimulera désormais l'auteur lui-même, qui conduira son double de l'enfance à l'âge adulte. Chroniques, comptes rendus, interviews, traductions presque simultanées dans plusieurs pays assureront à sa littérature une diffusion rapide et le succès.

En Roumanie, il avait été militant syndical, l'un des collaborateurs importants de la gazette România muncitoare (La Roumanie ouvrière), principale publication du mouvement socialiste de l'époque, et avait publié des articles entre 1906 et 1915. En 1919, il avait débuté en Suisse, en français, comme journaliste.

Le début littéraire ne le fera pas renoncer à son intérêt pour la vie sociale et politique. En 1924, il envoie de Paris plusieurs articles dont l'écho est considérable dans la presse roumaine, car leur auteur est auréolé de la gloire littéraire acquise en France. Quand il revient en Roumanie en 1925, après neuf ans d'absence, les autorités le suspectent d'être agent communiste chargé de missions secrètes. Sans être membre du Parti Communiste Français, il en était pourtant un «compagnon de route». Accueilli cordialement par beaucoup d'écrivains roumains, il est cependant violemment attaqué dans la presse, accusé de bolchevisme, mais aussi de fascisme.

En 1927, comme vice-président de l'Association française «Les Amis de l'U.R.S.S. », il est invité à Moscou, pour y participer aux festivités du dixième anniversaire de la Révolution d'Octobre 1917. $\mathrm{Au}$ début de 1928, il revient en U.R.S.S., cette fois à son propre compte et sans obligations officielles ou protocolaires. Il connaît ainsi directement la réalité de la "patrie prolétarienne », " la monstrueuse révélation », écrira-t-il déçu, comme d'autres désenchantés, Georges 
Duhamel (Voyage à Moscou, 1927), Hergé (Tintin au pays des Soviets, 1930) ou André Gide (Retour de l'U.R.S.S., 1936). Sa position bouleversera sa vie et éloignera de lui de nombreux amis, dont Romain Rolland, Jean-Richard Bloch et Henri Barbusse, sans en attirer de nouveaux. La presse communiste le qualifiera désormais de traître, de vendu au capitalisme, d'agent de la « Sigourantza », c'est-àdire de la police secrète roumaine. Il déclarera même dans la presse que « la terreur communiste est pire que celle fasciste ». Rejeté par les uns, il n'est pas admis par les autres.

Durant l'année 1928, deux manuscrits, Mes départs et Les Chardons du Baragan, remis par Istrati à la librairie Gallimard, respectivement à l'éditeur Bernard Grasset, sont publiés en son absence.

Les Chardons du Baragan s'ouvrent sur des images poétiques et se terminent sur des pages de misère et de sang. Le Baragan, vaste plaine désertique de la Valachie danubienne, rebelle à toute culture de la terre, ne se laisse féconder que par les chardons qui l'envahissent. Tous les ans, lorsque le Crivatz, le vent froid venu de Russie, se met à souffler, les chardons ploient sous sa force, et les boules épineuses, arrachées à leurs tiges, commencent à rouler en flocons compacts, couvrant l'immense étendue infertile de leurs vagues moutonneuses. Pour les enfants, c'est le plaisir des courses dans le vent, parmi les innombrables chardons. Pour l'homme, c'est la mauvaise graine qui se propage et s'implante dans le terrain, et contre laquelle il devra lutter.

Tout comme la canaille humaine: plus elle est inutile, et mieux elle sait se défendre (Istrati $1928: 13$ ).

La misère s'installe dans le Baragan. La pluie se déverse, détrempe le sol et pourrit les céréales. Les chevaux ne peuvent plus tirer les charrettes. Les hommes, enfoncés dans la boue jusqu'aux genoux, n'ont d'autre ressource que de porter sur leurs épaules les quelques gerbes rassemblées avec peine.

Cette misère, que les boyards, propriétaires des terres et maîtres des redevances, veulent ignorer, engendre la colère, car le paysan accepte la vie rude, le manque d'argent, mais non la faim. Or, pour 
payer ses dettes, il livre les produits agricoles qu'il a pu sauver du désastre et, durant tout le dur hiver, il aura le ventre creux, lui et sa famille. Il regimbe, mais il supporte encore son mal jusqu'à ce que, poussé à bout par les interventions brutales et répétées de la force publique, il se révolte. Les fusils de chasse, détournés de leur usage, tuent des hommes. L'horrible est déchaîné. La force restera à la loi, qui rétablira l'ordre sur les cadavres de beaucoup de paysans et les ruines de trois villages.

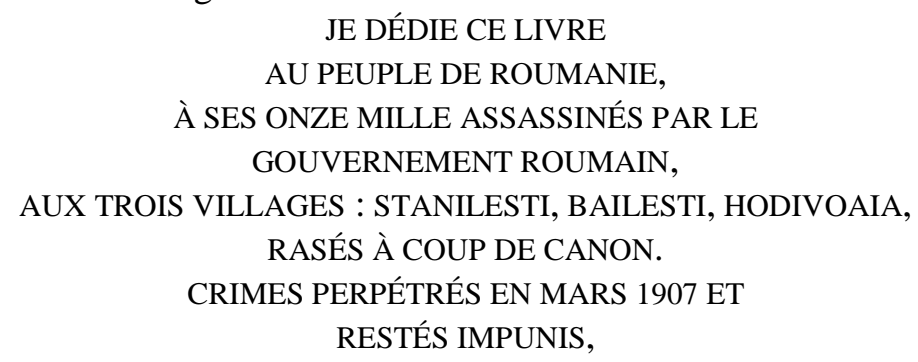

écrit l'auteur sur la dernière page de son livre, juste avant la cassette éditoriale, comme dans une sorte de post-dédicace.

Ce livre, peut-être le plus important de toute sa création, n'est pas seulement un cri de révolte. C'est aussi une «épopée de la faim» (Bataillon 1931: 27 ; Serres $1977:$ 198).

Sa problématique est inspirée de l'univers rural roumain du début $\mathrm{du} \mathrm{XX}^{\mathrm{e}}$ siècle. Il offre une trame épique riche en destinées, un horizon fictionnel-cosmique, social, psychologique-fortement symbolisé, une action principale et des actions subsidiaires en crescendo, une motivation architecturale intérieure et un ample dénouement tragique. Il est à la fois roman et pamphlet.

L'âge du narrateur se situe entre l'enfance et l'adolescence. Le roman résume les aventures de Mataké qui veut connaître le monde. Celui-ci se réduit, dans sa vision candide, à l'univers palpable, accessible, visible, mais aussi à l'univers caché, fabuleux, hyperbolique, qui est censé exister derrière lui. Son devenir sera représenté par une suite de révélations grâce auxquelles l'enfant s'ouvre au cosmos et à l'expérience ontologique. Le processus de 
cette prise de conscience commence par la première découverte, celle de la réalité physique où il est implanté, la campagne originaire infinie, espace qu'il perçoit dans sa réalité primordiale et sociale. Les autres révélations viendront lorsqu'il aura pris en possession cette réalité, et dissocié ses niveaux. Il découvrira l'inégalité sociale comme une indigence universelle, grâce aux paysans de la plaine du Baragan, éternellement soumis à leurs exploiteurs, les boyards. Il découvrira la pauvreté, dans la maison paternelle, au manoir de «Mademoiselle » (Doudouca), dans la maison de père Toma, le carrossier, et chez les habitants des Trois-Hameaux. Il connaîtra la joie du travail, mais aussi son prix qu'il fallait payer à l'époque, à côté de son père, comme vendeur de poisson et coupeur de bois, ensuite comme domestique à la cour hospitalière de «Mademoiselle» ou à celle de Costaké. Il découvrira et exploitera le rêve comme panacée à ses illusions existentielles. Il se trouvera affronté à la mort, d'abord à celle de sa mère, atténuée par la distance, puis à celle collective, à laquelle il assistera comme témoin involontaire et traumatisé. Il rencontrera partout la révolte, et y participera. Tout ce qu'il verra et éprouvera deviendra la réalité de sa conscience. Ses perceptions enfantines fulgurantes finiront dans des systématisations conscientes et cérébrales. Son long chemin durera autant que son enfance frustrée, et le mènera à la méditation sociale.

Au début, il prend acte de l'horizon géographique. La plaine qui s'offre à lui est vide, informe et élémentaire. Elle vit dans sa somnolence millénaire. Son immobilité archaïque rappelle un monstre qui suggère à l'enfant des visions terrifiantes. L'infini lui fait comprendre la solitude et même l'adversité :

Étendu, depuis l'éternité, sur toutes les terres que le soleil grille entre la dolente Yalomitsa et le Danube grognon, le Baragan est, durant le printemps et l'été, en guerre sournoise avec l'homme laborieux qu'il n'aime pas et auquel il refuse tout bien-être, sauf celui de se promener et de hurler (Istrati 1928 : 9).

Mais, curieusement, dans son profond primitivisme, cet espace émane une poésie rude, qui invite au recueillement. Il devient même un excellent médiateur du rêve et de l'évasion du quotidien austère. Son 
manque de limites acquiert ainsi une magnificence troublante, et inspire l'œil :

Un oiseau qui vole entre deux chaînes de montagnes, c'est une chose qui fait pitié. Sur le Baragan, le même oiseau emporte dans son vol la terre et ses lointains horizons. Allongé sur le dos, vous sentez l'assiette terrestre qui se soulève et monte vers le zénith. C'est la plus belle des ascensions que puisse faire le pauvre dépourvu de tout (Istrati $1928: 10-11$ ).

Même l'immensité grise des chardons qui obscurcissent le champ visuel - symboles de la pétrification archaïque et de la stérilité semble une explosion florale, dans la lumière pure des aubes :

Aussitôt je me sentis emporté, car le soleil, surgissant brusquement, jeta sur notre solitude sa gerbe de rayons aveuglants. Les milliers de chardons bourrus s'emplirent de diamants violacés, que j'allais toucher du doigt ou cueillir avec le bout de la langue [...] (Istrati $1928: 12$ ).

Mais, au-delà de l'horizon géographique et circonscrit à lui, Mataké découvrira l'horizon social. Le chantre des plaines lointaines est remplacé par le pamphlétaire. Les pages poétiques céderont la place aux longues dissertations sur la condition du paysan roumain à la veille de la première guerre mondiale. Mataké a un penchant évident à la généralisation à propos de sa communauté rurale. Les images sont sombres et non-différenciées. Les paysans sont d'une pauvreté invraisemblable. Ils passent leur vie à exploiter obstinément leurs petits lopins de terre presque stériles, battent leurs femmes et leurs bestiaux, mettent au monde beaucoup d'enfants, que, faute de pouvoir nourrir, ils abandonnent sur la plaine du Baragan, se laissent aller à l'ivrognerie, plutôt par manque de perspective que par hérédité. Le tableau suivant rappelle la stridence des généralisations zoliennes :

Chaque soir, sur les oulitza $a^{1}$ ténébreuses et défoncées, on pouvait voir une épouse, une mère, une sœur, traînant vers la chaumière un paysan qui s'écroulait tous les dix pas. La femme le suivait dans la boue, et recevait quelques bons coups. D'autres bons coups l'attendaient à la maison. Le lendemain matin amenait toujours le repentir, car l'homme au fond, n'était pas une brute. Il aidait alors au ménage, s'occupait du bétail, charriait de l'eau, et passait une bonne partie de la

${ }^{1}$ Rue étroite, ruelle (en roumain, dans le texte, au singulier et en orthographe française). 
journée à trier les ciocani $^{2}$, brûlant les uns, séchant les autres autour de la soba ${ }^{3}$. Les foyers, d'habitude propres, se transformaient en écuries, débordaient de boue et de moisissure jusque sur la table (Istrati 1928 : 174-175).

Istrati cède aussi à la tentation de peindre un portrait physique général de l'homme du Baragan, le cojan. Il est partout le même, accablé de la fatalité de la pauvreté, qui met son empreinte sur toutes les étapes de sa vie, en les mêlant et en les estompant :

C'étaient des pauvres diables, ces Vlachkans, pareils à ceux de chez nous, en Yalomitsa : maigres, la peau sur les os, le front plissé avant l'âge, l'œil terne, non rasés pendant des semaines. Sur leurs chemises, pendant jusqu'aux genoux, on ne pouvait plus compter les pièces. Leur pantalon n'était qu'un amas de lambeaux. Pieds nus, tête nue, vrais mendiants, ils me faisaient de la peine comme s'ils avaient tous été mes parents. Leurs femmes, la trentaine passée, semblaient des vieilles. Pressées par ce travail qui doit se faire rapidement, celles qui allaitaient abandonnaient leur bébé à quelque frérot, au milieu du maïs, où il hurlait jusqu'à s'étouffer. Des chiens allaient ronger les langes sales et lécher les visages (Istrati $1928: 157-158)$.

Sensible aux significations de la stratification sociale, Mataké continuera son exploration, et observera le détachement des jeunes par rapport aux tourments existentiels des parents. L'âge de l'initiation érotique les fait se soustraire aux préoccupations quotidiennes. Il évaluera le bien-être des élites du village en fonction de leur participation aux fêtes et aux noces. Il trouvera partout l'inflexibilité des boyards devant la pauvreté et les souffrances de la communauté. Le portrait monographique du paysan cojan tend à la stéréotypie, dont les traits sont le goût de la causerie, la sincérité primaire des sentiments, conformément au décor fruste, l'humilité infinie devant la dignité sociale, dérivée de la conscience tragique de la pauvreté, et cachant une révolte secrète. Tous les personnages du roman, y compris Mataké, correspondent plus au moins à ce portrait, dont ils sont souvent les nuances. Le père de Mataké est le cojan romanesque et vaporeux, sans esprit pratique, assez insolite dans la littérature roumaine, où le paysan vit par la vigueur primaire et la volonté de

${ }^{2}$ Épi de maïs sans grains (en roumain, dans le texte).

${ }^{3}$ Poêle ou cheminée (en roumain, dans le texte). 
parvenir dans la hiérarchie sociale. Il préfère à l'existence commune qui l'opprime la mélodie plaintive de la doïna ${ }^{4}$, où il se réfugie comme pour se soustraire aux déterminations sociales. Son portrait, l'un des plus consistants du livre, sera, par conséquent une métaphore suave et efféminée dans cet univers brutal et élémentaire :

[II] n'avait rien du mâle : c'était une douce femme, avec de grosses moustaches noires et des yeux profonds et langoureux, constamment posés sur sa flûte, d'où il tirait, avec ses doigts noueux, de douces mélodies qui retentissaient au loin et faisaient aboyer les chiens par les nuits silencieuses [...]. Le pauvre père ne relevait jamais une injure et supportait tout stoïquement. Esquissant un léger sourire, il s'en allait vers la Borcea, avec son bonnet pointu toujours rejeté sur la nuque, avec sa culotte en loques, toujours mal ficelée, ses opinci $i^{5}$ traînantes, son long cou et son merveilleux caval ${ }^{6}$, qui ne manquait pas, lui, de le venger de cette vie pitoyable et tristement belle (Istrati $1928: 23-24$ ).

Aucun des héros de Panaït Istrati n'est si sensible. La scène où il se disculpe de la mort du cheval, parce que dans son subconscient il s'en considère comme coupable, l'anime d'une tragique poésie, et l'approche de l'humanité des moujiks tolstoïens :

Ôtant sa caciula $^{7}$, mon père dit, en regardant le cadavre :

— Dieu m'est témoin que je ne l'ai pas fait souffrir. J'ai couru à trois portées de fusil pour lui chercher de l'eau ; l'herbe ne lui a pas manqué ; et de fouet, je n'en ai point. Si elle [la bête] est morte entre mes mains, que Dieu me pardonne, mais je n'y suis pour rien. Il se signa et fit une génuflexion, face à ce levant, d'où il était parti sans espoir (Istrati $1928: 54$ ).

Pour le rendre encore plus vivant, Istrati l'oppose constamment à Mataké, dans la vision duquel, peuplée de haïdouks ${ }^{8}$ et de colporteurs,

\footnotetext{
${ }^{4}$ Poésie lyrique spécifique au folklore roumain, qui exprime un sentiment de tristesse, de nostalgie, de révolte, d'amour, accompagnée d'habitude d'une mélodie adéquate (en roumain, dans le texte).

${ }^{5}$ Chaussure paysanne faite d'un morceau rectangulaire de cuir ou de caoutchouc, et serrée sur la plante des pieds par des lanières (en roumain, dans le texte).

${ }^{6}$ Grande flûte de berger (en roumain, dans le texte).

${ }^{7}$ Bonnet de fourrure (en roumain, dans le texte).

${ }^{8}$ Haïdouk, brigand justicier qui, révolté contre l'oppression, quittait sa maison et vivait dans le bois, isolé ou en bande, en pillant les riches et en aidant les pauvres (en roumain, dans le texte).
} 
le père apparaît comme une note discordante dans le paysage, contraire à la typologie commune des habitants du Baragan et, surtout, à ses rêves incendiés d'évasions grandioses.

Le visage féminin domine la figuration caractérologique d'Istrati. La paysanne du Baragan est volontaire et sensuelle, animée par la passion et l'esprit de sacrifice, la sincérité et la révolte. Elle est favorisée par la vision qui la transfigure, celui du personnagenarrateur, l'enfant qui sort peu à peu de son enfance. Elle est donc vue soit comme mère tendre et aimante, soit comme appât érotique. La mère de Mataké personnifie le culte de la maternité, et l'absence d'autorité ferme du père la fait cumuler des attributs volontaires, paternels. Elle fond, selon les circonstances, sa dureté dans une féminité protectrice. Elle est ennoblie par son amour passionné pour son mari, qu'elle défend comme une douce fatalité :

Je l'aime, mon petit... Dieu l'a fait ainsi et me l'a donné pour mari. Ce n'est pas sa faute, à lui, le pauvre homme ! (Istrati $1928: 25$ ).

Doudouca est aussi une passionnée, pour laquelle la vie tellurique finit avec les méandres heureux de l'amour. Elle s'impose avec une auréole légèrement légendaire qui imprime une atmosphère romantique au décor. Elle a vécu autrefois un drame amoureux, qu'elle remémore avec une volupté délibérée de souffrance. La douleur déchirante ressentie à la mort de son amant la jette dans une vie quasi-monacale qu'elle accepte comme un pont qui la relie à son passé heureux. De la véranda de son manoir en ruine perdu dans l'immensité du Baragan, elle revit sa jeunesse, et l'assimile à la vie même, par l'intermédiaire du rêve, provoqué et cultivé.

Toudoritza a une robustesse ostentatoire et une profondeur érotique nuancée. Elle est présentée par l'adolescent Mataké, ce qui explique son portrait langoureux. Elle aime impétueusement, mais l'homme qu'elle aime est tiraillé entre l'amour et l'argent. Son caractère évoluera vers la révolte. Son drame individuel passe au premier plan de l'intrigue, et est souvent considéré comme le seul noyau narratif du livre. 
Les enfants sont assez nombreux dans le roman. Leurs regards altèrent la réalité physique, et lui confèrent une dimension mythique. Ils reconnaissent le Baragan comme un royaume légendaire infini peuplé de contes de fée. L'horizon géographique qui s'ouvre à leur connaissance se colore grâce à leur vision, et se déforme dans le plan mythique. En fonction de l'angle de la lumière, de la saison ou du moment de la journée, la campagne leur suggère des visages anthropomorphiques étranges. Les choses et les gens acquièrent des contours fallacieux. Au-dessus de la plaine déserte plane un léger brouillard onirique, qui atténue le contingent strident et lui confère une patine archaïque.

L'altération mythique au niveau de l'horizon social est ressentie dans la narration lorsque les héros se mettent à rêver de transcender l'inertie de la pauvreté. Le besoin de rêver est d'ailleurs pour eux une seconde nécessité biologique, après la nourriture. Le rêve suspend la temporalité du personnage, et le plonge dans une réalité paradisiaque, projection mentale de ses options obsessionnelles. L'adolescent s'imaginera des fiançailles avec de pâles héroïnes en crinolines dentelées, bercées par le trot des chevaux nobiliaires, des rencontres légendaires avec les brigands justiciers des bois, des fuites de la maison, équivalant à l'entrée dans leur rang, des rituels fantastiques invoquant les dieux champêtres, et dont le point de départ est la ronde des chardons, portés par la bise.

La mythification de l'horizon moral de la narration se réalise par l'idéalisation des caractères. La mère de Mataké, par exemple, aime son mari avec une sorte de damnation fataliste. Doudouca a l'air d'une véritable princesse valaque, dévouée dans un désert sauvage et anhistorique au culte archaïque sépulcral de son fiancé entré dans les brumes légendaires. Le père de Mataké semble se superposer à un personnage folklorique, généreux et naïf, romanesque, modeste et maladroit, communiquant plutôt avec le monde zoomorphe qu'avec le monde des humains. La souffrance et la pauvreté suggèrent les ténèbres médiévales mal précisées dans leurs coordinations spatiotemporelles, stagnantes dans une durée ponctuée par l'arrivée et le départ des cigognes, la bise, les fêtes traditionnelles. 
Le devenir de Mataké est une initiation à la souffrance. La damnation est générale dans le roman, chaque personnage représentant un Job sui generis. Seul le père de Mataké la comprendra dans le sens évangélique, comme un calvaire, «Golgotha de la vie». Les autres personnages l'interpréteront du point de vue social, en l'appelant manque de terre, pauvreté, faim, inégalité, et s'évertueront à la détruire par la révolte. L'expérience de cette lutte confère du sens à l'existence de Mataké. Il connaîtra d'abord la souffrance, parallèle à son réveil à la vie cosmique et à son initiation. Il la rencontrera partout dans son aventure, et l'assimilera aux choses éternelles. Parfois il essaiera, mais en vain, de l'atténuer par le rêve.

Le chemin de Mataké, de la découverte de la souffrance à son approfondissement par la révolte, est aussi visible dans l'évolution sémantique du mot «chardons». Au début, ils n'ont qu'un sens purement ludique, «beaux comme de grands buis, nombreux comme les étoiles, charnus, crevant de sève » (Istrati 1928:39), incendiant le regard avec leur danse funambulesque, et invitant au rêve. Près d'eux, les grandes audaces de l'enfance semblent possibles. Leur frémissement et leur ondoiement sous le vent transforment le Baragan en un océan infini où les cimes empourprées par le couchant paraissent de vraies vagues en perpétuel mouvement.

L'entrée de Costaké dans le récit confère aux chardons une extension métaphorique. Leur sphère enveloppe une catégorie sociale qui, au niveau plus restreint de la communauté villageoise, démontre leur existence inféconde. C'est pourquoi ils deviendront les chardonsboyards, métaphore des dénivellements sociaux du monde du Baragan. Dans ce sens, le cadre social est toujours plus assimilé symboliquement à la campagne restée en friche, où les chardons règnent, malgré son infertilité.

L'incendie des chardons sera, dans le récit, la métaphore de la justice sociale. Le moment de l'éclat de la révolte paysanne est aussi fixé par un «immense embrasement » (Istrati 1928: 226).

La révolte sera présentée de l'angle exclusif de Mataké, qui assiste à son accumulation potentielle. Il est le sensible séismographe des douleurs étouffées, qui atteignent leur comble dans le village des 
Trois-Hameaux, parce qu'elle contraste avec le bien-être d'une petite couche privilégiée. Les autorités villageoises se profilent au fond, le maire, le gendarme, le pope, l'administrateur. De l'autre côté, les paysans pauvres, grâce auxquels Mataké devient conscient des souffrances et de l'imminence de la révolte. Celle-ci finira dans le sang. Mataké, son ami Brêche-Dent et Costaké, aveuglés par la fumée, cherchent leur chemin dans la nuit, les chardons courant après eux.

Les chaumières en flammes dispersaient à tous les vents leur toit de paille brûlante. On ne faisait plus attention aux cadavres qu'on heurtait à chaque pas, mais aux vivants qui s'accrochaient à nous et nous empêchaient de fuir (Istrati $1928: 231-232$ ).

L'aventure finit donc dramatiquement, dans l'inquiétude. Le chemin mène à la connaissance de la véritable souffrance du paysan, est auréolé par la révolte, mais reste suspendu devant une perspective toujours ouverte sur cette souffrance, sans autre solution que celle de la répétition du cycle. Un sens profond l'ennoblit pourtant : il s'ouvre de nouveau vers le monde.

Bibliographie

Bataillon, M. (1931): Le roman picaresque. Paris: La Renaissance du Livre.

Istrati, P. (1928): Les Chardons du Baragan. Paris: Grasset.

Serres, M. (1977): «Tintin ou le picaresque aujourd'hui », Critique, n 358, pp. 197207. 